\title{
Eficiência de sistemas de refrigeração sem uso de energia: experimentos a partir do sistema pot in pot
}

\section{Francisco Tiago Carvalho Silva ${ }^{1}$, Cleverson Ramon Carvalho Silva ${ }^{2}$ e Bernat Vinolas Prat $^{3}$}

${ }^{1}$ Programa de Pós-Graduação em Saúde, Sociedade \& Ambiente. Mestrado Profissional Interdisciplinar. Universidade Federal dos Vales do Jequitinhonha e Mucuri. Campus JK. Rodovia MGT-367, km 583, no 5000. Bairro Alto do Jacuba. Diamantina-MG, Brasil (CEP 39100-000).

${ }^{2}$ Instituto Federal de Educação, Ciência e Tecnologia do Sudeste de Minas Gerais. Campus Manhuaçu. Rodovia BR-116, km 589,8. Distrito Realeza. Manhuaçu-MG, Brasil (CEP 36905-000).

${ }^{3}$ Universidade Federal dos Vales do Jequitinhonha e Mucuri. Instituto de Ciência e Tecnologia. Campus JK. Rodovia MGT-367, km 583, n 5000. Bairro Alto do Jacuba. Diamantina-MG, Brasil (CEP 39100-000). E-mail: bernatvinolasprat@gmail.com.

Resumo. A situação de vulnerabilidade social e pobreza, somadas as dificuldades para conservação de frutas e legumes em alguns países levou ao desenvolvimento de um dispositivo alternativo denominado Pot in Pot, sistema similar a uma geladeira que dispensa o uso de energia elétrica. Voltado ao estudo deste sistema, o objetivo principal deste artigo é avaliar três sistemas Pot in Pot a fim de encontrar os aspectos-chave para melhoria da eficiência deste dispositivo. 0 referencial teórico que embasa este estudo aborda os conceitos de física aplicado aos experimentos e alguns tipos de equipamentos reportados na literatura. A metodologia fundamentou-se na realização de testes laboratoriais para avaliar a eficiência dos três sistemas Pot in Pot: sistema 1, vaso com água e sem tampa; sistema 2 , modelo original do Pot in Pot, ou seja, um vaso menor posto dentro de outro vaso maior e separados por areia umedecida e com tampa de pano umedecido; e o sistema 3 feito a partir do segundo, tendo como diferença construtiva o lixamento da área externa do vaso maior. A partir dos dados obtidos foi realizada a análise estatística. Os resultados indicaram que (a) o dispositivo, vaso com água, teve a menor eficiência de arrefecimento em relação demais experimentos, (b) os dispositivos analisados tiveram eficiências semelhantes a outras pesquisas desenvolvidas sobre o mesmo tema; (c) que o lixamento da área externa do vaso maior colaborou de forma significativa para a melhoria do funcionamento do dispositivo 3 , vaso lixado, sendo este o experimento com maior taxa de eficiência de arrefecimento.
Recebido $01 / 06 / 2020$

Aceito

$10 / 04 / 2021$

Disponível on line

$12 / 04 / 2021$

Publicado

$30 / 04 / 2021$

Acesso aberto

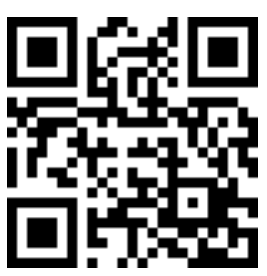

ORCID

(1) 0000-0001-9443-4966

Francisco Tiago

Carvalho Silva

ISSN 2359-1412/RBGAS-2020-0090/2021/8/18/12/195

Rev. Bras. Gest. Amb. Sustent.

http://revista.ecogestaobrasil.net 
Palavras-chave: Eficiência; Pot in pot; Resfriamento evaporativo.

\section{Abstract. Evaluation of the efficiency of non-energy refrigeration systems: Experiments from the Pot in Pot System.} The situation of social vulnerability and poverty, in addition to the difficulties in conserving fruits and vegetables in some countries, led to the development of an alternative device called Pot in Pot, a system similar to a refrigerator that dispenses with the use of electricity. Aimed at studying this system, the main objective of this article is to evaluate 3 Pot in Pot systems in order to find the key aspects for improving the efficiency of this device. The theoretical framework that supports this study addresses the concepts of physics applied to experiments and some types of equipment reported in the literature. The methodology was based on laboratory tests to assess the efficiency of the 3 Pot in Pot systems: system 1 , pot with water and without a lid; system 2 , original Pot in Pot model, that is, a smaller vase placed inside another larger vase and separated by moist sand and with a moist cloth cover; and system 3 made from the second, having as a constructive difference the sanding of the outer area of the larger vessel. From the data obtained, statistical analysis was performed. The results indicated that (a) the device, pot with water, had the lowest cooling efficiency in relation to other experiments, (b) the analyzed devices had efficiencies similar to other researchers developed on the same theme, (c) that the sanding of the outer area of the larger vessel contributed significantly to the improvement of the functioning of device 3, the sanded vessel, which is the experiment with the highest cooling efficiency rate.

Keywords: Efficiency; Pot in pot; Evaporative cooling.
D) 0000-0003-0985-8807

Cleverson Ramon

Carvalho Silva

(1) 0000-0002-0002-2811

Bernat Vinolas Prat

\section{Introdução}

A geração, transmissão e distribuição de energia elétrica alcançaram avanços significativos nas últimas décadas. Graças ao investimento estatal e do setor privado em pesquisa e desenvolvimento de novas tecnologias, o acesso à energia elétrica deixou de ser um privilégio, tornando-a um bem acessível para a maioria das pessoas (Van Valkenburgh e Neville, 1992). Entretanto, essa não é a realidade para grande parte da população pobre.

Conforme dados informados pelo Banco Mundial (G1, 2019), apesar dos avanços tecnológicos, em 2019, cerca de 840 milhões de pessoas ainda não têm acesso à energia elétrica, este valor corresponde a $10 \%$ da população mundial, a maior parte localizada na África Subsaariana. A falta de energia elétrica gera inúmeros malefícios, dentre eles, o não acesso a equipamento de refrigeração. Normalmente, os alimentos são armazenados em sistemas que necessitam de energia elétrica para o controle da temperatura e umidade relativa do ar.

A correta temperatura e a umidade relativa do ar são aspectos essenciais para a conservação de alimentos. Frutas e vegetais são perecíveis, e por este motivo, devem ser armazenados em ambientes com índice de temperaturas e umidade controladas. A baixa temperatura e a alta umidade possuem fundamental importância para inibir a proliferação microbiana e minimizar reações químicas que causam a deterioração dos alimentos, ou 
seja, ela é essencial tanto para manter as características do produto, quanto para aumentar sua vida útil (Mürmann et al., 2005; Basediya et al., 2013).

Considerando esse contexto e preocupado com as dificuldades locais da sua comunidade, Mohammed Bah Abba, professor nigeriano, desenvolveu uma solução para o armazenamento de frutas e legumes. 0 "pote em pote", originalmente pot in pot, é constituído de um vaso menor que é inserido em outro vaso maior, o espaço entre eles é preenchido por areia, a areia é molhada para possibilitar o resfriamento. 0 dispositivo é simples e eficiente para reduzir a temperatura em seu interior, em comparação com a temperatura ambiente, o que resultou na sua utilização em comunidades carentes de países da África (Barros Filho et al., 2011, p. 2).

Partindo da problemática aqui exposta, o objetivo geral deste artigo é avaliar três sistemas pot in pot a fim de encontrar aspectos-chaves para melhoria da eficiência destes dispositivos. Os objetivos específicos são:

1) analisar a eficiência de três tipos de dispositivos baseados no sistema Pot in Pot: o primeiro com água, o segundo vazio e o terceiro com vaso lixado;

2) avaliar se o aumento do volume de água eleva a eficiência do dispositivo;

3) avaliar se o aumento da porosidade, por meio do lixamento da área externa do vaso, eleva sua eficiência de resfriamento.

A presente pesquisa se justifica ao propor o aprimoramento do dispositivo por meio da avaliação dos aspectos-chave que melhoram sua eficiência de resfriamento. Outro fator é a popularização de uma solução simples de geladeira de baixo custo, que dispensa o uso energia elétrica. A ideia central desta pesquisa é colocar a ciência a serviço da população desfavorecida socioeconomicamente, por meio da divulgação e do aprimoramento do referido experimento.

Os conceitos físicos que permitem explicar o funcionamento do sistema pot in pot são o resfriamento evaporativo e a transferência de calor. 0 resfriamento evaporativo concerne-se a um processo adiabático, ou seja, transcorre sem trocas de calor externas ao mesmo. 0 efeito resultante de redução da temperatura pode ser explicado mediante conceitos de transferência de calor e conseguintes alterações de propriedades psicrométricas do ar, ressaltando-se a umidade relativa e temperaturas de bulbo seco e úmido, que se dão de forma interna. As limitações do resfriamento se estabelecem em função da diferença entre as temperaturas de bulbo seco e úmido e pela umidade relativa. 0 processo será mais eficiente tão maior for a diferença entre as citadas temperaturas juntamente a uma baixa condição de umidade relativa. Sinteticamente, o efeito de climatização é produzido ao se evaporar a água que é inserida ao processo. Essa mudança de estado é decorrente de transferência de calor entre o ar quente e água quando em contato, promovendo à mesma o calor para vaporização (Abreu et al., 1999; Dadhich et al., 2008).

De acordo com Gustafsson e Simson (2016) o movimento do ar em uma superfície faz com que a transferência de calor aumente devido a renovação do ar já aquecido pelo ar mais frio, desta forma, quanto maior for a diferença de temperatura do ar e do corpo mais calor poderá ser transferido. Este mesmo princípio ocorre na transferência de massa, a exemplo da água em uma superfície em contato com o ar, ela se distribui uniformemente pelo meio e vaporiza caso a umidade relativa seja mais baixa.

Da mesma forma, a água irá se distribuir uniformemente por um material desde que seja suficientemente poroso. A superfície externa do pot in pot será mantida úmida e a evaporação continuará até não restar mais água ou até que o ar tenha uma umidade relativa igual ou superior a do ambiente. Quando a água evapora de uma superfície, é necessário energia ou calor para transformar a água do estado líquido para o gasoso. Essa energia que é retirada da água ainda na superfície, fazendo como que a água diminua de 
temperatura e, portanto, resfria-se o corpo (superfície do vaso externo) (Gustafsson e Simson, 2016).

Desde aparição do primeiro dispositivo pot in pot para resfriamento evaporativo criado por Mohammed Bah Abba, outros dispositivos foram desenvolvidos e estão descritos a seguir. As variações em relação ao projeto original referem-se à utilização de outros materiais ou aplicação de dimensões diferentes, contudo, todos possuem o mesmo conceito físico de funcionamento (resfriamento evaporativo).

- Sistema de resfriamento evaporativo composto de parede externa de tijolos, câmara interna feita a partir de uma caixa metálica e separados por areia umedecida do mar, que funciona como meio para evaporar a água (Falayi and Jongbo, 2011).

- Sistema constituído de parede dupla de tijolos, preenchimento da espaço vazio com areia do leito do rio. Para tampa utilizou-se pedaços de bambu e para manter a câmara umedecida uso-se pulverizador de água (Dadhich et al., 2008).

- Três Sistemas feitos a partir da técnica pot in pot, os dispositivos foram testados em diferentes alturas para avaliar se uma melhor ventilação aumentava a eficiência dos sistemas. 0 estudo concluiu que o aumento da altura favorece a ventilação e consequentemente aumenta sua eficiência de arrefecimento (Yahaya et al., 2019).

- Um dispositivo onde foram testados diferentes materiais vegetais de preenchimento em substituição à areia para avaliar a melhora na eficiência de arrefecimento do dispositivo (Gunadasa et al., 2017).

- Ogle et al. (2016) analisaram o uso de vasos e outras técnicas para conservação de insulina. A pesquisa verificou a eficiência de resfriamento dos dispositivos estudados.

- Com base no modelo pot in pot, Guimarães et al. (2017) aplicaram o uso da técnica para melhorar o conforto térmico de uma residência. 0 estudo reproduziu um modelo reduzido para verificar a redução de temperatura interna. A pesquisa alcançou valores de redução de temperatura média de $3,8{ }^{\circ} \mathrm{C}$.

- Wiedenhefdt et al. (2013), verificaram o uso de isopor em substituição da areia no montagem do pot in pot. Nesta pesquisa o isopor demonstrou-se ineficiente.

Alguns estudos, como Yahaya et al. (2019) e Gunadasa et al. (2017) avaliaram aspectos da melhora da eficiência do dispositivo colocando o equipamento em lugares de melhor ventilação ou preenchendo a lacuna com materiais diferentes. Porém, não foram encontrados estudos realizados onde se objetive melhorar a eficiência dos dispositivos realizando uma alteração na superfície cerâmica do vaso exterior, de forma a aumentar a velocidade do processo do resfriamento evaporativo.

\section{Material e métodos}

\section{Local do estudo}

A pesquisa foi realizada no Laboratório de Construções Sustentáveis, do Mestrado Interdisciplinar Saúde, Sociedade e Ambiente (SaSA), no Campus JK, da Universidade Federal dos Vales do Jequitinhonha e Mucuri (UFVJM), em Diamantina, Minas Gerais. As coordenadas de referência são $18^{\circ} 12^{\prime} 10,0^{\prime \prime}$ S e $43^{\circ} 34^{\prime} 30,7^{\prime \prime}$ W, e altitude de 1.387 m. 


\section{Dimensionamento dos sistemas pot in pot}

Inicialmente foram adquiridos no comércio local dois vasos cerâmicos de tamanhos e dimensões diferentes:

- vaso externo (maior): $24 \mathrm{~cm} \mathrm{x} 18 \mathrm{~cm}$ x $17 \mathrm{~cm}$ ( $\varnothing$ superior, altura, $\emptyset$ inferior), com espessura da parede aproximada de 1,2 cm;

- vaso interno (menor): $17 \mathrm{~cm} \mathrm{x} 14 \mathrm{~cm}$ x $9 \mathrm{~cm}$ ( $\varnothing$ superior, altura, $\varnothing$ inferior), com espessura da parede aproximada de 1,2 cm.

- a espessura média da areia foi variável, na parte superior $2,3 \mathrm{~cm}$ e na inferior $2,8 \mathrm{~cm}$.

Os vasos comercializados geralmente possuem um furo em sua base, o primeiro passo foi fechá-lo com argamassa de cimento, outra alternativa é a cola epóxi. Com o furo devidamente fechado foi montado o primeiro sistema (Figura 1a) vaso maior com água e sem tampa. Posteriormente, o segundo dispositivo foi constituído pelo modelo original do pot in pot, ou seja, um vaso menor posto dentro de outro vaso maior e separados por areia umedecida e com tampa de pano umedecido, situação mantida pelo acréscimo diário de água, até o final do estudo (Figura 1b). 0 terceiro dispositivo foi feito a partir do segundo tendo como diferença construtiva o lixamento da área de externa do vaso maior, feito por meio da lixa grossa no 80 e frisos horizontais e verticais feitos a partir de um garfo de mesa em aço inox (Figura 1c).

(a)

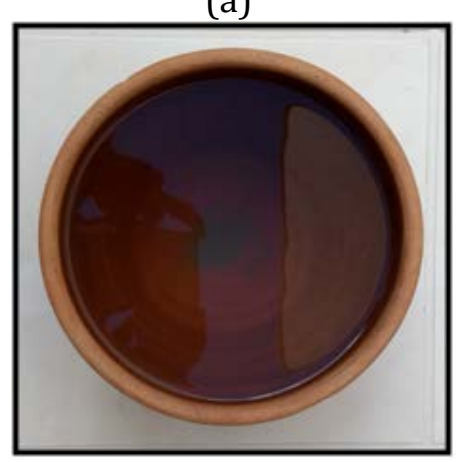

(b)

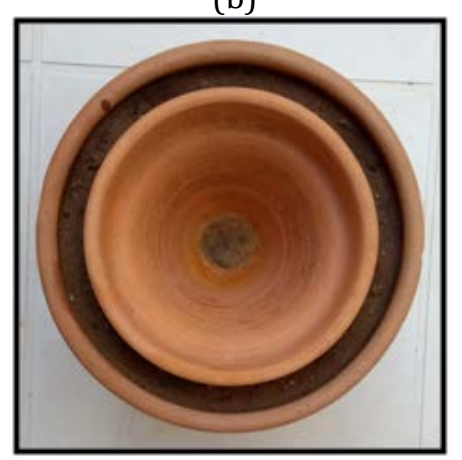

(c)

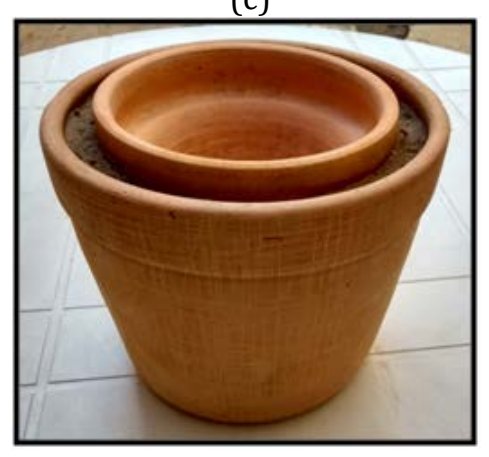

Figura 1. (a) Sistema 1 - Vaso com água; (b) Sistema 2 - Vaso vazio; (c) Sistema 3 - Vaso lixado.

\section{Instrumentos}

Para a aferição diária da temperatura $\left(\mathrm{T}^{\circ} \mathrm{C}\right)$ e a umidade relativa do ar foram os utilizados o termo-higrômetro digital (Hikari HK-T240) e termômetro portátil (Qualxtron - QX110). 0 sensor de temperatura foi instalado na parte interna central dos vasos e o sensor de temperatura ambiente e umidade relativa do ar foi instalado aproximadamente a $60 \mathrm{~cm}$ de distância de cada experimento.

\section{Análise dos dados}

Durante os três dias foram aferidas a temperatura e a umidade relativa do ar dos três sistemas, conforme a seguir:

1. Vaso com água (um vaso cerâmico com água e sem tampa), por meio de 36 medições; 
2. Vaso vazio (pot in pot modelo original), por meio de 42 medições;

3. Vaso lixado (pot in pot modelo original com vaso externo lixado), por meio de 45 medições.

Foi observado o comportamento de cada sistema em relação às situações diversas de temperatura e umidade relativa do ar (UR).

Segundo Carvalho Silva e Vinolas Prat (2020) para se calcular a eficiência de arrefecimento dos sistemas evaporativos não se deve observar apenas a diferença entre Temperatura interna e ambiente (Tin-Tamb), é importante verificar a máxima redução de temperatura que o sistema pode atingir, essa máxima redução de temperatura trata-se da temperatura de bulbo úmido (Tbu). Portanto, a eficiência de arrefecimento evaporativo foi calculada pela seguinte fórmula: $\mathrm{E}=(\mathrm{Tamb}-\mathrm{Tin}) /(\mathrm{TAmb}-\mathrm{Tbu})$.

A TAmb e Tin foram obtidas utilizando equipamentos de medição termohigrômetro e pelo termômetro portátil, a Tbu foi calculada pelo aplicativo AgaisPsicro (Agais, 2020).

Visando a atender aos objetivos propostos no estudo e de posse dos dados coletados, foram realizados testes estatísticos, conforme necessidade de cada análise. Os dados foram processados por meio do software Statistical Package for the Social Sciences (SPSS), versão 17.0, para Windows. Os dados coletados de $\left(\mathrm{T}^{\circ} \mathrm{C}\right.$ ) e de (UR) foram analisados por ANOVA e as médias foram comparadas utilizando-se o teste de Tukey com $\mathrm{p}<0.05$.

\section{Resultados}

Para obtenção dos dados, foram aferidas a temperatura e umidade relativa do ar de cada sistema analisado durante três dias, , cujos resultados encontram-se detalhados nas tabelas de 1 a 3.

Tabela 1. A comparação das eficiências do vaso com água.

\begin{tabular}{|c|c|c|c|c|c|}
\hline Horário & $\begin{array}{c}\text { Temperatura } \\
\text { interna }\end{array}$ & $\begin{array}{c}\text { Temperatura } \\
\text { ambiente }\end{array}$ & $\begin{array}{c}\text { Temperatura de } \\
\text { bulbo úmido }\end{array}$ & $\begin{array}{c}\text { Umidade } \\
\text { relativa }\end{array}$ & $\begin{array}{c}\text { E = (Tamb - Tin) } \\
\text { /(Tamb - Tbu) }\end{array}$ \\
\hline (HH:MM) & Tin $\left({ }^{\circ} \mathbf{C}\right)$ & Tamb $\left({ }^{\circ} \mathbf{C}\right)$ & \multicolumn{7}{|c|}{ Tbu ( $\left.{ }^{\circ} \mathbf{C}\right)$} & UR (\%) & E (\%) \\
\hline \multicolumn{7}{|c|}{$\mathbf{1 0}$ dia 09/01/2020 } \\
\hline $11: 00$ & 22,9 & 25,7 & 21,91 & 72 & $73,9 \%$ \\
\hline $11: 20$ & 22,7 & 25,1 & 21,07 & 70 & $59,6 \%$ \\
\hline $11: 40$ & 22,6 & 25,3 & 21,25 & 70 & $66,7 \%$ \\
\hline $12: 00$ & 22,6 & 25,4 & 21,34 & 70 & $69,0 \%$ \\
\hline $12: 20$ & 22,6 & 25,9 & 21,65 & 69 & $77,6 \%$ \\
\hline $12: 40$ & 22,5 & 26,0 & 21,59 & 68 & $79,4 \%$ \\
\hline $13: 00$ & 22,4 & 25,6 & 21,08 & 67 & $70,8 \%$ \\
\hline $13: 20$ & 22,3 & 25,3 & 20,66 & 66 & $64,7 \%$ \\
\hline $13: 40$ & 22,1 & 25,1 & 20,48 & 66 & $64,9 \%$ \\
\hline $14: 00$ & 22,1 & 24,6 & 20,04 & 66 & $54,8 \%$ \\
\hline $14: 20$ & 22,1 & 25,3 & 20,51 & 65 & $66,8 \%$ \\
\hline
\end{tabular}


Tabela 1. Continuação.

\begin{tabular}{|c|c|c|c|c|c|}
\hline Horário & $\begin{array}{c}\text { Temperatura } \\
\text { interna }\end{array}$ & $\begin{array}{c}\text { Temperatura } \\
\text { ambiente }\end{array}$ & $\begin{array}{l}\text { Temperatura de } \\
\text { bulbo úmido }\end{array}$ & $\begin{array}{l}\text { Umidade } \\
\text { relativa }\end{array}$ & $\begin{array}{c}\text { E }=(\text { Tamb }- \text { Tin }) \\
/(\text { Tamb }- \text { Tbu })\end{array}$ \\
\hline (HH:MM) & $\operatorname{Tin}\left({ }^{\circ} \mathrm{C}\right)$ & Tamb $\left({ }^{\circ} \mathrm{C}\right)$ & Tbu $\left({ }^{\circ} \mathrm{C}\right)$ & UR (\%) & E (\%) \\
\hline \multicolumn{6}{|c|}{2 dia 10/01/2020 } \\
\hline $11: 20$ & 22,3 & 25,9 & 19,96 & 58 & $60,6 \%$ \\
\hline $11: 40$ & 22,2 & 26,2 & 20,05 & 57 & $65,0 \%$ \\
\hline $12: 00$ & 22,2 & 26,0 & 20,20 & 59 & $65,5 \%$ \\
\hline $12: 20$ & 22,0 & 25,9 & 19,80 & 57 & $63,9 \%$ \\
\hline $12: 40$ & 21,8 & 25,9 & 19,64 & 56 & $65,5 \%$ \\
\hline $13: 00$ & 21,7 & 25,9 & 19,64 & 56 & $67,1 \%$ \\
\hline $13: 20$ & 21,7 & 26,2 & 19,89 & 56 & $71,3 \%$ \\
\hline $13: 40$ & 21,7 & 26,2 & 19,89 & 56 & $71,3 \%$ \\
\hline $14: 00$ & 21,7 & 26,5 & 19,98 & 55 & $73,6 \%$ \\
\hline $14: 20$ & 21,6 & 26,7 & 19,99 & 54 & $76,0 \%$ \\
\hline \multicolumn{6}{|c|}{30 dia $11 / 01 / 2020$} \\
\hline 09:40 & 20,7 & 24,5 & 18,45 & 56 & $62,8 \%$ \\
\hline $10: 00$ & 20,6 & 23,8 & 17,85 & 56 & $53,8 \%$ \\
\hline $10: 20$ & 20,4 & 23,8 & 18,15 & 58 & $60,2 \%$ \\
\hline $10: 40$ & 20,2 & 23,8 & 18,30 & 59 & $65,5 \%$ \\
\hline $11: 00$ & 20,1 & 24,3 & 18,73 & 59 & $75,4 \%$ \\
\hline $11: 20$ & 20,2 & 24,5 & 18,91 & 59 & $76,9 \%$ \\
\hline $11: 40$ & 20,2 & 25,0 & 18,87 & 56 & $78,3 \%$ \\
\hline $12: 00$ & 20,3 & 25,2 & 18,89 & 55 & $77,7 \%$ \\
\hline $12: 20$ & 20,4 & 25,2 & 18,57 & 53 & $72,4 \%$ \\
\hline $12: 40$ & 20,4 & 25,5 & 18,82 & 53 & $76,3 \%$ \\
\hline $13: 00$ & 20,5 & 25,2 & 18,57 & 53 & $70,9 \%$ \\
\hline $13: 20$ & 20,5 & 25,2 & 18,73 & 54 & $72,6 \%$ \\
\hline $13: 40$ & 20,5 & 25,9 & 19,48 & 55 & $84,1 \%$ \\
\hline $14: 00$ & 20,6 & 25,9 & 19,32 & 54 & $80,5 \%$ \\
\hline $14: 20$ & 20,6 & 25,9 & 19,32 & 54 & $80,5 \%$ \\
\hline & & Desvio Padrão & $7,45 \%$ & Média Geral & $69,9 \%$ \\
\hline
\end{tabular}

Tabela 2. A comparação das eficiências-vaso vazio.

\begin{tabular}{|c|c|c|c|c|c|}
\hline Horário & $\begin{array}{c}\text { Temperatura } \\
\text { interna }\end{array}$ & $\begin{array}{c}\text { Temperatura } \\
\text { ambiente }\end{array}$ & $\begin{array}{c}\text { Temperatura de } \\
\text { bulbo úmido }\end{array}$ & $\begin{array}{c}\text { Umidade } \\
\text { relativa }\end{array}$ & $\begin{array}{c}\mathbf{E}=\mathbf{( T a m b}-\text { Tin) } \\
/(\text { Tamb - Tbu) }\end{array}$ \\
\hline (HH:MM) & Tin $\left({ }^{\circ} \mathbf{C}\right)$ & Tamb $\left({ }^{\circ} \mathbf{C}\right)$ & Tbu $\left({ }^{\circ} \mathbf{C}\right)$ & UR $(\%)$ & E (\%) \\
\hline \multicolumn{7}{|c|}{$\mathbf{1 0}$ dia $\mathbf{1 3 / 0 1 / 2 0 2 0}$} \\
\hline $10: 00$ & 22,9 & 26,2 & 20,17 & 56,70 & $54,7 \%$ \\
\hline $10: 20$ & 22,5 & 26,5 & 19,77 & 53,70 & $59,4 \%$ \\
\hline $10: 40$ & 22,1 & 26,5 & 19,77 & 53,70 & $65,4 \%$ \\
\hline $11: 00$ & 21,9 & 26,7 & 19,75 & 52,60 & $69,1 \%$ \\
\hline
\end{tabular}


Tabela 2. Continuação.

\begin{tabular}{|c|c|c|c|c|c|}
\hline Horário & $\begin{array}{c}\text { Temperatura } \\
\text { interna }\end{array}$ & $\begin{array}{l}\text { Temperatura } \\
\text { ambiente }\end{array}$ & $\begin{array}{l}\text { Temperatura de } \\
\text { bulbo úmido }\end{array}$ & $\begin{array}{l}\text { Umidade } \\
\text { relativa }\end{array}$ & $\begin{array}{c}\text { E }=(\text { Tamb }- \text { Tin }) \\
/(\text { Tamb }- \text { Tbu })\end{array}$ \\
\hline (HH:MM) & $\operatorname{Tin}\left({ }^{\circ} \mathrm{C}\right)$ & Tamb $\left({ }^{\circ} \mathrm{C}\right)$ & Tbu $\left({ }^{\circ} \mathrm{C}\right)$ & UR (\%) & E (\%) \\
\hline $11: 20$ & 21,7 & 26,9 & 20,21 & 54,30 & $77,7 \%$ \\
\hline $11: 40$ & 21,5 & 27,0 & 19,89 & 51,90 & $77,4 \%$ \\
\hline $12: 00$ & 21,5 & 26,9 & 19,94 & 52,70 & $77,6 \%$ \\
\hline $12: 20$ & 21,6 & 26,9 & 20,12 & 53,80 & $78,2 \%$ \\
\hline $12: 40$ & 21,6 & 27,0 & 20,16 & 53,50 & $78,9 \%$ \\
\hline $13: 00$ & 21,7 & 27,1 & 20,41 & 54,50 & $80,7 \%$ \\
\hline $13: 20$ & 21,8 & 27,2 & 20,19 & 52,70 & $77,0 \%$ \\
\hline $13: 40$ & 21,8 & 27,2 & 20,14 & 52,40 & $76,5 \%$ \\
\hline $14: 00$ & 21,8 & 27,3 & 19,98 & 51,00 & $75,1 \%$ \\
\hline $14: 20$ & 21,8 & 27,4 & 20,29 & 52,30 & $78,8 \%$ \\
\hline \multicolumn{6}{|c|}{$2^{\circ}$ dia $14 / 01 / 2020$} \\
\hline 09:40 & 22,5 & 26,1 & 20,60 & 61,0 & $65,5 \%$ \\
\hline $10: 00$ & 22,1 & 25,6 & 20,17 & 61,0 & $64,5 \%$ \\
\hline $10: 20$ & 21,9 & 25,5 & 20,16 & 63,5 & $67,4 \%$ \\
\hline $10: 40$ & 21,8 & 25,8 & 20,48 & 61,9 & $75,2 \%$ \\
\hline $11: 00$ & 21,6 & 25,7 & 20,58 & 63,1 & $80,1 \%$ \\
\hline $11: 20$ & 21,5 & 26,0 & 20,30 & 59,6 & $78,9 \%$ \\
\hline $11: 40$ & 21,4 & 26,2 & 20,63 & 60,6 & $86,2 \%$ \\
\hline $12: 00$ & 21,5 & 26,5 & 20,65 & 59,1 & $85,5 \%$ \\
\hline $12: 20$ & 21,7 & 26,8 & 20,89 & 59,0 & $86,3 \%$ \\
\hline $12: 40$ & 21,7 & 26,8 & 20,84 & 58,7 & $85,6 \%$ \\
\hline $13: 00$ & 21,8 & 27,0 & 20,95 & 58,3 & $86,0 \%$ \\
\hline $13: 20$ & 21,9 & 27,1 & 20,94 & 57,7 & $84,4 \%$ \\
\hline $13: 40$ & 22,0 & 27,1 & 20,92 & 57,6 & $82,5 \%$ \\
\hline $14: 00$ & 22,0 & 27,2 & 20,94 & 57,2 & $83,1 \%$ \\
\hline $14: 20$ & 22,0 & 27,3 & 20,91 & 56,5 & $82,9 \%$ \\
\hline \multicolumn{6}{|c|}{ 3o dia 15/01/2020 } \\
\hline $10: 20$ & 21,9 & 23,6 & 20,70 & 77,2 & $58,6 \%$ \\
\hline $10: 40$ & 21,7 & 23,6 & 20,75 & 77,6 & $66,7 \%$ \\
\hline $11: 00$ & 21,6 & 23,8 & 20,80 & 76,6 & $73,3 \%$ \\
\hline $11: 20$ & 21,5 & 24,2 & 20,80 & 73,9 & $79,4 \%$ \\
\hline $11: 40$ & 21,6 & 24,9 & 21,09 & 71,4 & $86,6 \%$ \\
\hline $12: 00$ & 21,7 & 25,2 & 21,22 & 70,4 & $87,9 \%$ \\
\hline $12: 20$ & 21,7 & 25,4 & 21,22 & 69,2 & $88,5 \%$ \\
\hline $12: 40$ & 21,8 & 25,5 & 21,37 & 69,6 & $89,6 \%$ \\
\hline $13: 00$ & 21,8 & 25,6 & 21,38 & 69,0 & $90,0 \%$ \\
\hline $13: 20$ & 21,9 & 25,7 & 21,45 & 68,9 & $89,4 \%$ \\
\hline $13: 40$ & 22 & 25,8 & 21,57 & 69,1 & $89,8 \%$ \\
\hline
\end{tabular}


Tabela 2. Continuação.

\begin{tabular}{|c|c|c|c|c|c|}
\hline Horário & $\begin{array}{c}\text { Temperatura } \\
\text { interna }\end{array}$ & $\begin{array}{c}\text { Temperatura } \\
\text { ambiente }\end{array}$ & $\begin{array}{c}\text { Temperatura de } \\
\text { bulbo úmido }\end{array}$ & $\begin{array}{c}\text { Umidade } \\
\text { relativa }\end{array}$ & $\begin{array}{c}\mathbf{E}=\mathbf{( T a m b}-\text { Tin) } \\
/(\text { Tamb - Tbu) }\end{array}$ \\
\hline (HH:MM) & Tin $\left({ }^{\circ} \mathbf{C}\right)$ & Tamb $\left({ }^{\circ} \mathbf{C}\right)$ & Tbu $\left({ }^{\circ} \mathbf{C}\right)$ & UR $(\%)$ & E (\%) \\
\hline $14: 00$ & 22 & 25,7 & 21,32 & 68 & $84,5 \%$ \\
\hline $14: 20$ & 21,9 & 25,1 & 20,94 & 69,1 & $76,9 \%$ \\
\hline \multicolumn{2}{r|}{} & Desvio Padrão & $\mathbf{9 , 1 6 \%}$ & Média Geral & $\mathbf{7 8 , 1 \%}$ \\
\hline
\end{tabular}

Tabela 3. A comparação das eficiências do vaso lixado.

\begin{tabular}{|c|c|c|c|c|c|}
\hline Horário & $\begin{array}{c}\text { Temperatura } \\
\text { interna }\end{array}$ & $\begin{array}{c}\text { Temperatura } \\
\text { ambiente }\end{array}$ & $\begin{array}{l}\text { Temperatura de } \\
\text { bulbo úmido }\end{array}$ & $\begin{array}{l}\text { Umidade } \\
\text { relativa }\end{array}$ & $\begin{array}{c}E=(\text { Tamb }- \text { Tin }) \\
/(\text { Tamb }- \text { Tbu })\end{array}$ \\
\hline (HH:MM) & $\operatorname{Tin}\left({ }^{\circ} \mathrm{C}\right)$ & $\operatorname{Tamb}\left({ }^{\circ} \mathrm{C}\right)$ & Tbu $\left({ }^{\circ} \mathrm{C}\right)$ & UR (\%) & E (\%) \\
\hline \multicolumn{6}{|c|}{ 1o dia 13/01/2020 } \\
\hline $09: 40$ & 21,3 & 23,3 & 20,57 & 78,3 & $73,3 \%$ \\
\hline $10: 00$ & 21,3 & 22,9 & 20,27 & 78,9 & $60,8 \%$ \\
\hline $10: 20$ & 21,2 & 22,5 & 20,06 & 80,2 & $53,3 \%$ \\
\hline $10: 40$ & 21,0 & 22,6 & 20,58 & 83,5 & $79,2 \%$ \\
\hline $11: 00$ & 20,9 & 22,6 & 20,70 & 84,4 & $89,5 \%$ \\
\hline $11: 20$ & 20,7 & 22,2 & 20,15 & 83,1 & $73,2 \%$ \\
\hline $11: 40$ & 20,7 & 22,8 & 20,46 & 81,1 & $89,7 \%$ \\
\hline $12: 00$ & 20,7 & 23,3 & 20,62 & 78,7 & $97,0 \%$ \\
\hline $12: 20$ & 20,7 & 23,5 & 20,65 & 77,5 & $98,2 \%$ \\
\hline $12: 40$ & 20,8 & 23,6 & 20,82 & 78,1 & $100,7 \%$ \\
\hline $13: 00$ & 20,8 & 23,7 & 20,82 & 77,4 & $100,7 \%$ \\
\hline $13: 20$ & 20,9 & 23,8 & 20,88 & 77,2 & $99,3 \%$ \\
\hline $13: 40$ & 20,9 & 23,8 & 20,95 & 77,7 & $101,8 \%$ \\
\hline $14: 00$ & 21,0 & 23,9 & 20,96 & 77,1 & $98,6 \%$ \\
\hline $14: 20$ & 21,0 & 24,0 & 20,95 & 76,3 & $98,4 \%$ \\
\hline \multicolumn{6}{|c|}{$2^{\circ}$ dia 19/01/2020 } \\
\hline 09:40 & 20,7 & 22,7 & 20,10 & 79,0 & $76,9 \%$ \\
\hline $10: 00$ & 20,7 & 22,8 & 20,07 & 78,1 & $76,9 \%$ \\
\hline $10: 20$ & 20,7 & 22,9 & 20,09 & 77,5 & $78,3 \%$ \\
\hline $10: 40$ & 20,7 & 23,0 & 20,13 & 77,1 & $80,1 \%$ \\
\hline $11: 00$ & 20,7 & 22,8 & 20,32 & 80,0 & $84,7 \%$ \\
\hline $11: 20$ & 20,7 & 23,0 & 20,30 & 78,4 & $85,2 \%$ \\
\hline $11: 40$ & 20,7 & 23,2 & 20,37 & 77,5 & $88,3 \%$ \\
\hline $12: 00$ & 20,7 & 23,3 & 20,38 & 76,9 & $89,0 \%$ \\
\hline $12: 20$ & 20,8 & 23,4 & 20,45 & 76,7 & $88,1 \%$ \\
\hline $12: 40$ & 20,8 & 23,5 & 20,48 & 77,0 & $89,4 \%$ \\
\hline $13: 00$ & 20,8 & 23,5 & 20,42 & 75,8 & $87,7 \%$ \\
\hline $13: 20$ & 20,8 & 23,6 & 20,55 & 76,1 & $91,8 \%$ \\
\hline
\end{tabular}


Tabela 3. Continuação.

\begin{tabular}{|c|c|c|c|c|c|}
\hline Horário & $\begin{array}{c}\text { Temperatura } \\
\text { interna }\end{array}$ & $\begin{array}{l}\text { Temperatura } \\
\text { ambiente }\end{array}$ & $\begin{array}{l}\text { Temperatura de } \\
\text { bulbo úmido }\end{array}$ & $\begin{array}{l}\text { Umidade } \\
\text { relativa }\end{array}$ & $\begin{array}{c}\text { E = (Tamb }- \text { Tin }) \\
/(\text { Tamb }- \text { Tbu })\end{array}$ \\
\hline (HH:MM) & $\operatorname{Tin}\left({ }^{\circ} \mathrm{C}\right)$ & Tamb $\left({ }^{\circ} \mathrm{C}\right)$ & Tbu $\left({ }^{\circ} \mathrm{C}\right)$ & UR (\%) & E (\%) \\
\hline $13: 40$ & 20,9 & 23,6 & 20,46 & 75,4 & $86,0 \%$ \\
\hline $14: 00$ & 20,9 & 23,7 & 20,66 & 76,2 & $92,1 \%$ \\
\hline $14: 20$ & 21,0 & 23,7 & 20,59 & 75,7 & $86,8 \%$ \\
\hline \multicolumn{6}{|c|}{ 3o dia 20/01/2020 } \\
\hline 09:40 & 20,5 & 22,9 & 20,51 & 80,7 & $100,4 \%$ \\
\hline $10: 00$ & 20,5 & 23,0 & 20,42 & 79,3 & $96,9 \%$ \\
\hline $10: 20$ & 20,6 & 23,1 & 20,29 & 77,6 & $89,0 \%$ \\
\hline $10: 40$ & 20,6 & 23,2 & 20,22 & 76,4 & $87,2 \%$ \\
\hline $11: 00$ & 20,7 & 23,3 & 20,31 & 76,4 & $87,0 \%$ \\
\hline $11: 20$ & 20,7 & 23,4 & 20,34 & 75,9 & $88,2 \%$ \\
\hline $11: 40$ & 20,7 & 23,5 & 20,31 & 75,0 & $87,8 \%$ \\
\hline $12: 00$ & 20,7 & 23,6 & 20,41 & 75,1 & $90,9 \%$ \\
\hline $12: 20$ & 20,8 & 23,7 & 20,41 & 74,4 & $88,1 \%$ \\
\hline $12: 40$ & 20,8 & 23,7 & 20,40 & 74,3 & $87,9 \%$ \\
\hline $13: 00$ & 20,8 & 23,8 & 20,52 & 74,5 & $91,5 \%$ \\
\hline $13: 20$ & 20,9 & 23,9 & 20,55 & 74,1 & $89,6 \%$ \\
\hline $13: 40$ & 20,9 & 24,0 & 20,63 & 74,0 & $92,0 \%$ \\
\hline $14: 00$ & 21,0 & 23,9 & 20,65 & 74,8 & $89,2 \%$ \\
\hline $14: 20$ & 21,0 & 23,9 & 20,96 & 77,1 & $98,6 \%$ \\
\hline & & Desvio Padrão & $9,95 \%$ & Média Geral & $88,0 \%$ \\
\hline
\end{tabular}

Na Tabela 4 observamos a análise estatística descritiva da variável eficiência para os três sistemas. 0 sistema 3 onde a superfície do vaso maior foi lixada, para testar o aumento do processo de resfriamento evaporativo, foi a que obteve valores mais altos em relação a média e mediana das eficiências.

Tabela 4. Análises estatísticas descritivas.

\begin{tabular}{|c|c|c|c|c|}
\hline \multicolumn{3}{|l|}{ Grupo } & Estatística & Erro Padrão \\
\hline \multirow{10}{*}{ Vaso água } & \multicolumn{2}{|l|}{ Média } & 0,6989 & 0,0124 \\
\hline & \multirow{2}{*}{ 95\% Intervalo de Confiança para Média } & Limite inferior & 0,6737 & \\
\hline & & Limite superior & 0,724 & \\
\hline & \multicolumn{2}{|l|}{$5 \%$ da média aparada } & 0,7005 & \\
\hline & \multicolumn{2}{|l|}{ Mediana } & 0,7085 & \\
\hline & \multicolumn{2}{|l|}{ Variância } & 0,006 & \\
\hline & \multicolumn{2}{|l|}{ Desvio Padrão } & 0,07439 & \\
\hline & \multicolumn{2}{|l|}{ Mínimo } & 0,54 & \\
\hline & \multicolumn{2}{|l|}{ Máximo } & 0,84 & \\
\hline & \multicolumn{2}{|l|}{ Intervalo } & 0,3 & \\
\hline
\end{tabular}


Tabela 4. Continuação.

\begin{tabular}{|c|c|c|c|c|}
\hline \multicolumn{3}{|l|}{ Grupo } & Estatística & Erro Padrão \\
\hline \multirow{10}{*}{ Vaso vazio } & \multicolumn{2}{|l|}{ Média } & 0,7814 & 0,01413 \\
\hline & \multirow{2}{*}{ 95\% Intervalo de Confiança para Média } & Limite inferior & 0,7528 & \\
\hline & & Limite superior & 0,8099 & \\
\hline & \multicolumn{2}{|l|}{ 5\% da média aparada } & 0,7867 & \\
\hline & \multicolumn{2}{|l|}{ Mediana } & 0,789 & \\
\hline & \multicolumn{2}{|l|}{ Variância } & 0,008 & \\
\hline & \multicolumn{2}{|l|}{ Desvio Padrão } & 0,09157 & \\
\hline & \multicolumn{2}{|l|}{ Mínimo } & 0,55 & \\
\hline & \multicolumn{2}{|l|}{ Máximo } & 0,9 & \\
\hline & \multicolumn{2}{|l|}{ Intervalo } & 0,35 & \\
\hline \multicolumn{3}{|l|}{ Grupo } & Estatística & Erro Padrão \\
\hline \multirow{10}{*}{ Vaso lixado } & \multicolumn{2}{|l|}{ Média } & 0,8798 & 0,01483 \\
\hline & \multirow{2}{*}{ 95\% Intervalo de Confiança para Média } & Limite inferior & 0,8499 & \\
\hline & & Limite superior & 0,9097 & \\
\hline & \multicolumn{2}{|l|}{ 5\% da média aparada } & 0,8886 & \\
\hline & \multicolumn{2}{|l|}{ Mediana } & 0,89 & \\
\hline & \multicolumn{2}{|l|}{ Variância } & 0,01 & \\
\hline & \multicolumn{2}{|l|}{ Desvio Padrão } & 0,09951 & \\
\hline & \multicolumn{2}{|l|}{ Mínimo } & 0,53 & \\
\hline & \multicolumn{2}{|l|}{ Máximo } & 1,02 & \\
\hline & \multicolumn{2}{|l|}{ Intervalo } & 0,49 & \\
\hline
\end{tabular}

Na Tabela 5 é demonstrada a Análise ANOVA de comparação de médias da variável eficiência dos três sistemas mediante o teste de Tukey com $\mathrm{p}<0,05$. Comparando todas as médias se observa que o grau de significância é 0 . 0 teste Tukey HSD mostrou então que estatisticamente a média das eficiências coletadas dos três experimentos são significativamente diferentes. Pode-se afirmar que os diferentes experimentos realizados tiveram influência na alteração da eficiência dos dispositivos. Este fato observa-se de forma gráfica na Figura 2, onde se mostra o boxplot da variável estudada (eficiência) no caso dos três experimentos.

Tabela 5. Análise ANOVA.

\begin{tabular}{|c|c|c|c|c|c|c|}
\hline \multicolumn{7}{|c|}{ Comparações múltiplas } \\
\hline \multicolumn{7}{|c|}{ Variável dependente: Todos } \\
\hline \multicolumn{7}{|l|}{ Tukey HSD } \\
\hline \multirow[b]{2}{*}{ (I) Grupo } & \multirow[b]{2}{*}{ (J) Grupo } & \multirow[b]{2}{*}{$\begin{array}{l}\text { Diferença } \\
\text { média (I-J) }\end{array}$} & \multirow{2}{*}{$\begin{array}{c}\text { Erro } \\
\text { Padrão }\end{array}$} & \multirow[b]{2}{*}{ Sig. } & \multicolumn{2}{|c|}{ Intervalo de Confiança 95\% } \\
\hline & & & & & $\begin{array}{c}\text { Limite } \\
\text { inferior }\end{array}$ & $\begin{array}{c}\text { Limite } \\
\text { superior }\end{array}$ \\
\hline \multirow{2}{*}{ Vaso água } & Vaso vazio & $-0,08252^{*}$ & 0,02045 & 0,000 & $-0,1311$ & $-0,0340$ \\
\hline & Vaso lixado & $-0,18092^{*}$ & 0,02014 & 0,000 & $-0,2287$ & $-0,1331$ \\
\hline \multirow{2}{*}{ Vaso vazio } & Vaso agua & $0,08252^{*}$ & 0,02045 & 0,000 & 0,0340 & 0,1311 \\
\hline & Vaso lixado & $-0,09840^{*}$ & 0,01932 & 0,000 & $-0,1442$ & $-0,0525$ \\
\hline \multirow{2}{*}{ Vaso lixado } & Vaso agua & $0,18092^{*}$ & 0,02014 & 0,000 & 0,1331 & 0,2287 \\
\hline & Vaso vazio & $0,09840^{*}$ & 0,01932 & 0,000 & 0,0525 & 0,1442 \\
\hline
\end{tabular}




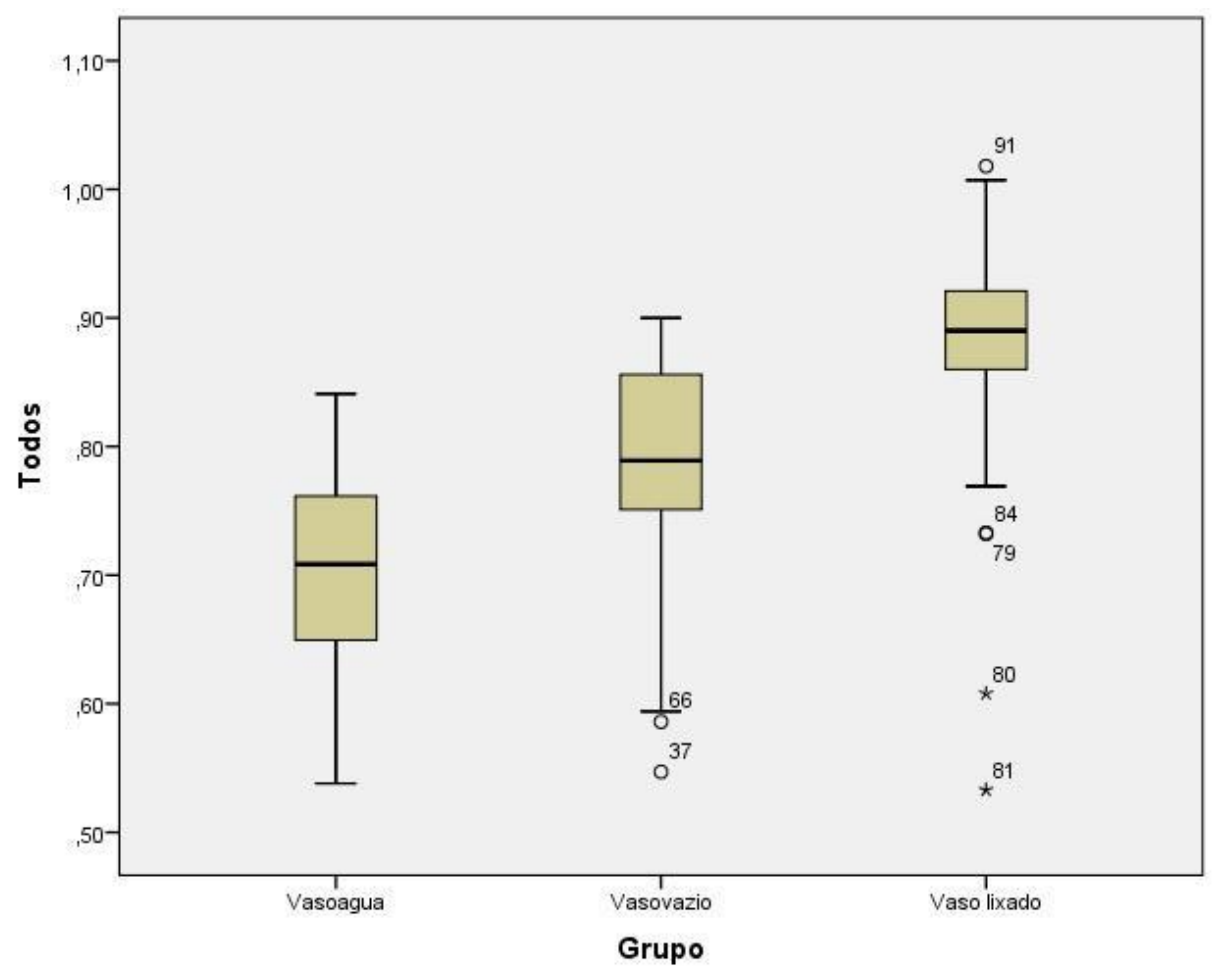

Figura 2. Boxplot (gráfico de caixa).

\section{Discussão}

\section{Comparativo das eficiências de arrefecimento}

0 dispositivo 1, vaso com água, foi o que teve o menor valor de média geral da eficiência de arrefecimento, 70.8\%, esse menor valor ocorreu, provavelmente, pelo grande volume de água a ser resfriado pelo equipamento.

0 dispositivo 2, vaso vazio, trata-se do modelo original do pot in pot, o mesmo obteve média geral da eficiência de arrefecimento de 78.9\%, achados semelhantes foram encontrados nas pesquisa de Yahaya et al. (2019) e Gunadasa et al. (2017).

0 dispositivo 3, vaso lixado, trata-se do modelo original do pot in pot tendo como diferencial o lixamento da superfície externa, o que possibilitou o aumento da área de superfície externa do vaso maior e, consequentemente, o aumento da área de contato com o ar do ambiente. Este equipamento obteve a maior média geral da eficiência de arrefecimento, $89,0 \%$ valor superior ao demais dispositivos e superior aos valores encontrados em outras pesquisa realizadas, a exemplo das mencionadas na Tabela 6.

\section{Aspectos-chave para o bom funcionamento dos dispositivos}

Segundo Odesola e Onyebuchi (2009), os aspectos-chave para a melhoria da eficiência de arrefecimento dos sistemas pot in pot são a umidade relativa, temperatura, movimento do ar e a área de superfície. 
Tabela 6. A comparação das eficiências de arrefecimento de diferentes pesquisas.

\begin{tabular}{|l|c|c|c|c|c|c|c|}
\hline \multicolumn{7}{|c|}{ Média das leituras } \\
\hline Autores & Localização & $\begin{array}{c}\text { UAmb } \\
(\mathbf{\%})\end{array}$ & $\begin{array}{c}\mathbf{T A m b} \\
\left({ }^{\circ} \mathbf{C}\right)\end{array}$ & $\begin{array}{c}\text { Tin } \\
\left({ }^{\circ} \mathbf{C}\right)\end{array}$ & $\begin{array}{c}\text { Tbu } \\
\left({ }^{\circ} \mathbf{C}\right)\end{array}$ & $\begin{array}{c}\text { Eficiência de } \\
\text { arrefecimento (\%) }\end{array}$ & Observações \\
\hline Yahaya et al. (2019) & Nigeria & 53,5 & 31,3 & 22,2 & 20,0 & $80,0 \%$ & $\begin{array}{c}\text { A um metro } \\
\text { de altura }\end{array}$ \\
\hline Dadhich et al. (2008) & Índia & 49,7 & 27,7 & 23,9 & 20,1 & $50,6 \%$ & $\begin{array}{c}\text { Fevereiro } \\
\text { (carregado) }\end{array}$ \\
\hline Ogle et al. (2016) & Índia & 35,3 & 40,2 & 32,1 & 27,3 & $62,8 \%$ & $\begin{array}{c}3 \text { meses mais } \\
\text { quentes }\end{array}$ \\
\hline Gunadasa et al. (2017) & Índia & - & - & - & - & $69,0 \%$ & Com areia \\
\hline
\end{tabular}

Fonte: Carvalho Silva e Vinolas Prat (2020, p. 38).

A umidade relativa do ar (UR) influência de forma significativa o funcionamento dos dispositivos, segundo Abreu et al. (1999) quanto menor a UR maior será a absorção do ar, ou seja, quanto mais seco for o ar maior será a evaporação da água. Conforme pode-se observar nas tabelas de 1 a 3, o mesmo pôde ser verificado no experimento realizado, as melhores eficiências foram obtidas nos horários com menores valores de UR.

As maiores temperaturas favorecem a evaporação da água, deste modo, espaços com temperaturas elevadas serão propícios para o resfriamento evaporativo (Odesola e Onyebuchi, 2009). Conforme verificado, pode-se afirmar que as maiores eficiências foram alcançadas nos horários com maiores temperaturas associadas à baixa umidade relativa do ar.

Sobre o movimento do ar, entende-se como aspecto importante para renovação do ar úmido no local por ar seco, caso não haja ventilação, o ar úmido não será substituído e a taxa de evaporação irá diminuir (Odesola e Onyebuchi, 2009). Na referida pesquisa não foi avaliada a influência deste aspecto em relação a eficiência dos equipamentos analisados.

Segundo Carvalho Silva e Vinolas Prat (2020) a evaporação é influenciada diretamente pela área de superfície, para esses autores quanto maior for a área de superfície a partir do qual a água pode evaporar, maior será a taxa de evaporação, ou seja, quanto maior a área de contato com o ar que circunda, melhor será o resfriamento neste local (p. 36 Esse foi o aspecto-chave de maior relevância deste estudo, pois o aumento da porosidade da superfície externa do vaso maior, por meio do lixamento, colaborou de forma significativa para o bom funcionamento do sistema, como pode ser comprovado pelos resultados extraídos na Tabela 3.

\section{Conclusões}

Com os ensaios realizados concluímos que:

1) 0 ar se resfria mais rápido do que o mesmo volume de água, este é o provável motivo para que o dispositivo, vaso com água, ter a menor eficiência de arrefecimento em relação aos demais experimentos.

2) Foi possível reproduzir os experimentos seguindo a técnica pot in pot. Os dispositivos tiveram eficiências semelhantes a outras pesquisas desenvolvidas sobre o mesmo tema.

3) 0 lixamento da área externa do vaso maior colaborou de forma significativa para o bom funcionamento do sistema 3, vaso lixado, sendo o experimento com maior taxa de eficiência de arrefecimento. 
Esta pesquisa investigou o aprimoramento do dispositivo por meio da avaliação dos aspectos-chave que melhoram sua eficiência de resfriamento, e buscou a popularização de uma solução simples de geladeira de baixo custo, que dispensa o uso energia elétrica. A ideia central desta pesquisa foi colocar a ciência a serviço da população desfavorecida socioeconomicamente, por meio da divulgação e do aprimoramento do referido experimento.

\section{Agradecimentos}

À Coordenação de Aperfeiçoamento de Pessoal de Nível Superior (CAPES); ao Conselho Nacional de Desenvolvimento Científico e Tecnológico (CNPq); à Pró-Reitoria de Pesquisa e Pós-Graduação (PRPPG), da Universidade Federal dos Vales do Jequitinhonha e Mucuri (UFVJM); ao Programa de Pós-Graduação em Saúde, Sociedade e Ambiente (PPGSaSA/UFVJM); ao Instituto de Ciência e Tecnologia (ICT/UFVJM); à Fundação Diamantinense de Apoio ao Ensino, Pesquisa e Extensão (FUNDAEPE); e à Fundação de Amparo à Pesquisa do Estado de Minas Gerais (FAPEMIG).

\section{Conflito de interesses}

Os autores declaram não haver conflito de interesses.

\section{Referências}

Abreu, P. G.; Abreu, V. M. N.; Mazzuco, H. Uso do resfriamento evaporativo (adiabático) na criação de frangos de corte. Corcórdia: Embrapa Suínos e Aves, 1999.

Agais. Agaispsicro: propriedades psicrométricas do ar. Agais. 2020. Disponível em: <http://agais.com/?pg=toolbox/spreadsheet_psicrometria>. Acesso em: 21 abr. 2020.

Barros Filho, J.; Bombati, T. A. V.; Bortoloti, J. A.; Cavalhieri, L.; Veraszto, E. V.; Camargo, J. T. F. Geladeira do deserto: uma iniciação científica forjada nas aulas de termodinâmica de um Curso de Engenharia Ambiental. Anais do XXXIX Congresso Brasileiro de Educação em Engenharia, Blumenau, 2011.

Basediya, A. L.; Samuel, D. V. K.; Beera, V. Evaporative cooling system for storage of fruits and vegetables: A review. Journal of Food Science and Technology, v. 50, n. 3, p. 429-442, 2013.

Carvalho Silva, F. T.; Vinolas Prat, B. Sistemas de refrigeração sem utilização de energia elétrica: uma análise da produção científica sobre dispositivos pot in pot. Revista Científica Multidisciplinar Núcleo do Conhecimento, v. 5, p. 21-42, 2020.

Dadhich, S. M.; Dadhich, H.; Verma, R. C. Comparative study on storage of fruits and vegetables in evaporative cool chamber and in ambient. International Journal of Food Engineering, v. 4, n. 1, 2008. https://doi.org/10.2202/1556-3758.1147

Falayi, F. R.; Jongbo, A. O. Development of metal-in-wall evaporative cooling system for storing perishable agricultural produce in a tropical environment. Journal of Agricultural Engineering and Technology, v. 19, n. 1, p. 35-45, 2011.

G1. Mais de 800 milhões de pessoas no mundo não têm acesso a energia elétrica, diz Banco Mundial. 2019. Disponível em: <https://g1.globo.com/economia/noticia/2019/05/22/ mais-de-800-milhoes-de-pessoas-no-mundo-nao-tem-acesso-a-energia-eletrica-dizbanco-mundial.ghtml>. Acesso em: 21 abr. 2020. 
Guimarães, M. S.; Silva, L. A.; Dias, P. S.; Gomes, R. C. C. Novo modelo de alvenaria visando ao resfriamento do ambiente interno. Revista Mirante, v. 10, n. 2, p. 108-118, 2017.

Gunadasa, H. L. C. K.; Awanthi, M. G. G.; Rupasinghe, C. P. Utilization of different plant based waste materials as alternatives to sand in zeer pot refrigerator. Journal of Engineering Research and General Science, v. 5, n. 2, p. 191-196, 2017.

Gustafsson, K.; Simson, H. An experimental study on an evaporative cooler for hot rural areas. Estocolmo: KTH School of Industrial Engineering and Management, 2016. (Tese de bacharelado).

Mürmann, L.; Mallmann, C. A.; Dilkin, P. Temperaturas de armazenamento de alimentos em estabelecimentos comerciais na Cidade de Santa Maria, RS. Acta Scientiae Veterinariae, v. 33, n. 3, p. 309, 2005.

Odesola, I. F.; Onyebuchi, O. A review of porous evaporative cooling for the preservation of fruits and vegetables. The Pacific Journal of Science and Technology, v. 10, n. 2, p. 935-941, 2009.

Ogle, G. D.; Abdullah, M.; Mason, D.; Januszewski, A. S.; Besançon, S. Insulin storage in hot climates without refrigeration: temperature reduction efficacy of clay pots and other techniques. Diabetic Medicine, v. 33, n. 11, p. 1544-1553, 2016. https://doi.org/10.1111/ dme.13194

Van Valkenburgh, N.; Neville, Inc. Eletricidade Básica. Rio de Janeiro: Ao livro Técnico, 1992. v. 1.

Wiedenhefdt, C. R.; Afonso, C. R.; Souza, É. C.; Selbach, D. J.; Freygang, C. C. Geladeira sem eletricidade. Anais do Salão de Ensino, Pesquisa e Extensão do IFRS Campus Canoas, 2013. Disponível em: <http://anaissaloes.canoas.ifrs.edu.br/index.php/enpex/article/view/ 171>. Acesso em: 23 set. 2019.

Yahaya, S. A.; Akande, K. A.; Raji, A. O.; Abdulrahman, K. O., Enagi, I. I. Effect of set-up heights on the performance of pot-in-pot cooling system for storing food and drugs at ambient temperature. FUOYE Journal of Engineering and Technology, v. 4, n. 1, p. 131-134, 2019. https://doi.org/10.46792/fuoyejet.v4i1.322

Informação da Licença: Este é um artigo Open Access distribuído sob os termos da Licença Creative Commons Attribution, que permite uso irrestrito, distribuição e reprodução em qualquer meio, desde que a obra original seja devidamente citada. 